ALEA, Lat. Am. J. Probab. Math. Stat. 18, 617-634 (2021)

\title{
A Doob-Meyer decomposition under model ambiguity: the case of compactness
}

\author{
Erick Treviño-Aguilar \\ Instituto de Matemáticas, Unidad Cuernavaca \\ Universidad Nacional Autónoma de México \\ Av. Universidad s/n, Cuernavaca Morelos \\ CP 62210, México. \\ E-mail address: erick.trevino@im.unam.mx
}

\begin{abstract}
We consider families of equivalent probability measures $\mathcal{Q}$ with a property related to concepts known in the literature under different names such as rectangularity or multiplicative stability. For the problems considered in this paper such a property yields dynamical consistency. We prove under a weak-compactness assumption with general filtrations and continuous processes that all semimartingales have an additive decomposition as the sum of a predictable non-decreasing process and a universal local supermartingale, by this concept we mean a process that is a local supermartingale with respect to each element of $\mathcal{Q}$. We also show that processes having a supermartingale property with respect to a superadditive nonlinear conditional expectation associated to the family $\mathcal{Q}$ are always semimartingales under weak-compactness. These results are relevant in stochastic optimization problems including optimal stopping under model ambiguity.
\end{abstract}

\section{Introduction}

The celebrated Doob-Meyer decomposition expresses a (local) supermartingale as the difference of a (local) martingale and a predictable non decreasing process. The structure of a supermartingale is in this way completely characterized and historically, it was instrumental in achieving a general theory of stochastic integration. The ubiquity of supermartingales gives a prominent role to the Doob-Meyer decomposition in applied contexts. In optimal stopping, value processes are supermartingales and their non decreasing parts characterize optimal stopping times. In mathematical finance, asset prices are modeled by a vector-valued semimartingale process and the capital generated by admissible investment strategies are supermartingales under appropriate equivalent changes of probability measures.

Received by the editors June 7th, 2019; accepted August 18th, 2020.

2010 Mathematics Subject Classification. 60G07,60G40,60G44,60H30.

Key words and phrases. Doob-Meyer Decomposition, Model ambiguity, Optimal stopping, Supermartingales, Semimartingales. 
In optimal stopping a reward process is represented by a stochastic process defined in a filtered base. Typically, its probabilistic properties, such as finite dimensional distributions, are uniquely determined when a unique a priori probability measure is fixed. A meaningful situation in which it is inconvenient to take a unique probability measure comes from economics. In decision theory, risk and model risk lead to differentiated behaviors as illustrated by the famous Ellsberg's Paradox; see Ellsberg (1961). Under risk, or in more probabilistic terms, uncertainty, one lies in the recipe just depicted of fixing a unique probability measure. Under model risk, also known as model ambiguity or Knight uncertainty, one considers a family of probability measures $\mathcal{Q}$ and each element is treated as a possible model from which the "correct distribution" is approximated. Then, for each model there is an expectation and value process of optimal stopping, the Snell envelope. A reasonable procedure to blend together all such quantifications is already provided in Epstein and Schneider (2003) axiomatic framework of dynamically consistent robust utility functionals. It consists on taking a conservative point of view and on each assessment always consider the worst possibility; in Remark 2.2 below, we make this point more precise. Hence, it is necessary to move from a expectation with respect to a unique probability measure to a superadditive non linear operator denoted by $\mathbf{E}^{\downarrow}$ arising as an infimum over the complete family of probability measures $\mathcal{Q}$. The value process of optimal stopping under model ambiguity is the so-called lower Snell envelope. It is defined as the infimum of all the Snell envelopes with respect to each element of $\mathcal{Q}$. Under dynamical consistency, it has a supermartingale property with respect to the operator $\mathbf{E}^{\downarrow}$ which we present more formally after this section in equation (2.2). A natural question is about the structure of processes having such a supermartingale property. Not just formally on considering the Doob-Meyer decomposition but also relevant in the context from which it was motivated, optimal stopping under model ambiguity. It is possible for an $\mathbf{E}^{\downarrow}$-supermartingale not to be a semimartingale; see Treviño-Aguilar (2017). Moreover, even if an $\mathbf{E}^{\downarrow}$-supermartingale is a semimartingale, an analogous structure extending in all generality the Doob-Meyer decomposition fails. This was shown in Treviño-Aguilar (2017) and here we will give a more systematic presentation of this fact.

Its time to describe our goal in this paper. We present a "positive" result for $\mathbf{E}^{\downarrow}$-supermartingales. More precisely, we give general conditions under which an $\mathbf{E}^{\downarrow}$ supermartingale has a generalized Doob-Meyer decomposition as the sum of a non decreasing process and a 'universal' $\mathcal{Q}$-supermartingale, by this we mean a process being a (local) supermartingale with respect to any probability measure in $\mathcal{Q}$. There are two main conditions and all refer to the class $\mathcal{Q}$ and not to the particular process. The first is a stability property which leads to dynamical consistency, also known as time consistency, of the operator $\mathbf{E}^{\downarrow}$. The second is weak-compactness of associated densities with respect to a reference probability measure. In a particular but generic specification of the family $\mathcal{Q}$, we illustrate how our condition of weak-compactness is optimal. There are two theorems which combinated prove our claim. In the first, Theorem 3.4, we show that any semimartingale can be compensated to be a universal $\mathcal{Q}$-supermartingale. In Theorem 3.6 we show that an $\mathbf{E}^{\downarrow}$-supermartingale is a supermartingale for some probability measure in $\mathcal{Q}$, showing in particular that it is a semimartingale. 
There are two classes of results in the literature we are aware of, that can be seen as extensions-of/related-to the Doob-Meyer decomposition, in the sense we are discussing here of taking into account a family of probability measures and not just a fixed probability measure. We start with the celebrated Optional Decomposition Theorem (ODT) which plays a fundamental role in stochastic finance. The ODT provides a decomposition in terms of a stochastic integral and $\mathcal{Q}$ is the family of local martingale measures of a semimartingale, interpreted as asset prices; see El Karoui and Quenez (1995); Kramkov (1996); Föllmer and Kramkov (1997); Föllmer and Kabanov (1998). In the most interesting case of incomplete markets, the family is far from being weakly compact and it applies only to what here we call universal $\mathcal{Q}$ supermartingales. Cheng and Riedel (2013) study optimal stopping problems under ambiguity. Building on Peng (1999, Theorem 3.3 and Corollary 3.12), they also establish a generalized version of the Doob-Meyer decomposition. Their method is based on Backward Stochastic Differential Equations on Brownian filtrations. None of the results in the present paper are covered by the literature just mentioned.

After this introduction the paper is organized as follows. In Section 2 we fix notation and introduce the main concepts to be used throughout the paper. In Section 3 we present the main results of the paper postponing their proofs to Sections 6 and 7. In Section 4 we introduce the concept of "finite interaction" emphasizing the role of Girsanov's tranformations to achieve the goal of the paper. In Section 5 we confirm and illustrate the general results of the paper in a concrete specification of the family $\mathcal{Q}$ with more explicit decompositions.

\section{Notations and setting}

Now we introduce some notation. For $\mathbb{T}>0$ a positive real number, we fix a stochastic base $\left(\Omega, \mathcal{F}, \mathbb{F}=\left\{\mathcal{F}_{t}\right\}_{0 \leq t \leq \mathbb{T}}, \mathbb{P}\right)$. We assume that the filtration $\mathbb{F}$ satisfies the usual conditions of right continuity and completeness. We assume that the probability measure $\mathbb{P}$ is $0-1$ on $\mathcal{F}_{0}$. Expectation with respect to $\mathbb{P}$ is simply denoted by $E[\cdot]$. Equality of random variables always means $\mathbb{P}$-a.s. equality. The family of $\mathbb{F}$-stopping times with values in the interval $[0, \mathbb{T}]$ is denoted by $\mathcal{T}$. The predictable $\sigma$-algebra defined on the set $\Omega \times[0, \mathbb{T}]$ is generated by the family of $\mathbb{F}$-adapted, continuous stochastic processes. This $\sigma$-algebra is usually denoted by $\mathcal{P}$; see e.g., Jacod and Shiryaev (2003, Section 2 a). For a predictable process $\xi$ and a semimartingale $S$ we denote by $\xi \cdot S$ its stochastic integral. Without further mention, we work in this paper with continuous semimartingales and denote by $\langle\cdot, \cdot\rangle$ the predictable covariation process of two semimartingales. The quadratic variation of a local martingale $M$ is denoted by $\langle M\rangle$. Our main topic in this paper deals with equivalent changes of probability measures and so we make repeated use of densities, to the extend that we systematically identify the probability measure with its density process. In particular, we define densities by Doléans-Dade stochastic exponential defined as the solution to the stochastic differential equation $d Z=Z d M$ where $M$ is a local martingale. In our setting, the process $M$ is a continuous local martingale and the solution to the stochastic differential equation, denoted by $\mathcal{E}(M)$ is explicitly given by $\exp \left\{M-\frac{1}{2}\langle M\rangle\right\}$. Let $L^{\infty}$ be the class of random variables which are essentially bounded.

For a process $V$ of finite variation we denote by $V=V^{+}-V^{-}$the decomposition arising from Hahn decomposition of measures, where $V^{+}$and $V^{-}$are non decreasing 
processes. We also denote by $a^{+}$the positive part of a number but this shall not cause confusion, since we never take the positive part of a finite variation process.

The next concept introduces our first requirement for a family of probability measures $\mathcal{Q}$.

Definition 2.1. Let $\tau \in \mathcal{T}$ be a stopping time and $Q_{1}$ and $Q_{2}$ be probability measures equivalent to $\mathbb{P}$. The probability measure defined through

$$
Q_{3}(A):=E_{Q_{1}}\left[Q_{2}\left[A \mid \mathcal{F}_{\tau}\right]\right], A \in \mathcal{F}_{\mathbb{T}}
$$

is called the pasting of $Q_{1}$ and $Q_{2}$ in $\tau$.

A family of probability measures $\mathcal{Q}$ is stable under pasting or simply stable if every $Q \in \mathcal{Q}$ is equivalent to $\mathbb{P}$, and if for each $Q_{1}$ and $Q_{2}$ in $\mathcal{Q}$ and any stopping time $\tau \in \mathcal{T}$, the pasting of $Q_{1}$ and $Q_{2}$ in $\tau$ is an element of $\mathcal{Q}$.

Definition 2.1 is a formulation presented in Föllmer and Schied (2004). It is essentially related to the concept of rectangularity or $m$ - stability (from multiplicative stability); see Delbaen (2006). The family of equivalent martingale measures is stable under pasting, and this property is crucial for the analysis of the upper and lower prices of American options; see Föllmer and Schied (2004). The stability concept appears also in the problem of representing time consistent risk measures; see e.g., Föllmer and Penner (2006) for details and references.

Here and in the sequel we denote by $L^{1}(Q)$ the space of random variables which are integrable with respect to a probability measure $Q$. For $\tau \in \mathcal{T}$, we denote by $L^{0}\left(\mathcal{F}_{\tau}\right)$ the space of finite valued $\mathcal{F}_{\tau}$-measurable functions. Let $\mathbf{E}^{\downarrow}\left[\cdot \mid \mathcal{F}_{\tau}\right]$ be the non-linear conditional expectation defined by

$$
\mathbf{E}^{\downarrow}\left[\cdot \mid \mathcal{F}_{\tau}\right]:=\operatorname{essinf}_{Q \in \mathcal{Q}} E_{Q}\left[\cdot \mid \mathcal{F}_{\tau}\right] .
$$

An $\mathbb{F}$-adapted process $\left\{X_{t}\right\}_{0 \leq t \leq \mathbb{T}}$ is a $\mathbf{E}^{\downarrow}$-supermartingale if for each pair of stopping times $\tau, \theta \in \mathcal{T}$ with $\mathbb{P}(\tau \geq \theta)=1$ we have $\inf _{Q \in \mathcal{Q}} E_{Q}\left[\left|X_{\tau}\right|\right]<\infty$, and

$$
\mathbf{E}^{\downarrow}\left[X_{\tau} \mid \mathcal{F}_{\theta}\right] \leq X_{\theta} .
$$

Remark 2.2. Some examples of $\mathbf{E}^{\downarrow}$-supermartingales are

(1) universal $\mathcal{Q}$-local-supermartingales defined as semimartingales which are local supermartingales with respect to each $Q \in \mathcal{Q}$. For simplicity we will just say $\mathcal{Q}$-supermartingales.

(2) Value processes of optimal stopping under model ambiguity from the point of view of having a right. More precisely, for $\mathrm{H}$ an adapted process, its lower Snell envelope is the "value process" of optimal stopping with respect to $\mathbf{E}^{\downarrow}$; see Föllmer and Schied (2004) for a systematic treatment in discrete time. Thus, it is a stochastic process $U^{\downarrow}$ such that for $t \in[0, \mathbb{T}]$, it is equal to

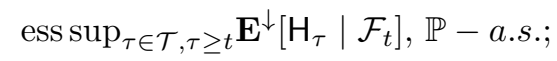

see Treviño-Aguilar (2012).

(3) Value processes of optimal stopping under model ambiguity from the point of view of having an obligation. The upper Snell envelope is the "value process" of optimal stopping with respect to the non linear expectation

$$
\operatorname{ess}_{\sup } \operatorname{su}_{Q \in \mathcal{Q}} E_{Q}\left[\cdot \mid \mathcal{F}_{\tau}\right] \text {; }
$$

see Föllmer and Kramkov (1997) and Föllmer and Schied (2004). 
In these three examples the stability of the family $\mathcal{Q}$ is essential for the analysis of the solution.

\section{Main results}

In this section we present the main results of the paper. In order to facilitate the lecture we include the proofs in separate sections.

Let $\mathcal{Q}$ be a family of probability measures. In this section we assume the properties of the next assumption. We denote by $\sigma\left(L^{1}, L^{\infty}\right)$ the weak topology on the Banach space $L^{1}$. Recall that $\sigma\left(L^{1}, L^{\infty}\right)$ has as subbase the family of sets $\left\{x \in L^{1}|| l(x-a) \mid \leq \epsilon\right\}$ for $\epsilon>0, a \in L^{1}$, and $l$ a continuous linear functional. For a presentation of weak topologies in $L^{p}$ spaces and Dunford-Pettis characterization of weak compactness see e.g., Bogachev (2007, Sections 4.7(ii) and 4.7(iv)).

Assumption 3.1. Let $\mathcal{Q}$ be a stable family of probability measures all equivalent to $\mathbb{P}$. Assume $\mathbb{P} \in \mathcal{Q}$ and the family of densities $\left\{\frac{d Q}{d \mathbb{P}}\right\}_{Q \in \mathcal{Q}}$ is $\sigma\left(L^{1}, L^{\infty}\right)$-compact. Let $\mathcal{N}$ be the class of all local martingales $N$ such that $\mathcal{E}(N)$ is the density process of a probability measure in $\mathcal{Q}$. We assume $\mathcal{N}$ is convex.

Remark 3.2. Note that for any $Z \in \mathcal{Q}$ the stochastic logarithm defined as the solution of the stochastic differential equation $d L=\frac{1}{Z} d Z$ is such that $\mathcal{E}(L)=Z$; see Revuz and Yor (1999, Proposition VIII (1.6)). As a consequence, a family satisfying Assumption 3.1 can always be represented as $\mathcal{Q}=\{\mathcal{E}(N) \mid N \in \mathcal{N}\}$.

Definition 3.3. A $\mathbb{P}$-local-martingale $M$ has a $\mathcal{Q}$-compensator if there exists a continuous non-decreasing process $B$ such that $M-B$ is a $\mathcal{Q}$-supermartingale.

A semimartingale $S$ has a $\mathcal{Q}$-compensator if its continuous local martingale part $S^{c}$ has a $\mathcal{Q}$-compensator.

The next theorem is our first main result. The proof is given in Section 6 .

Theorem 3.4. Under Assumption 3.1 a $\mathbb{P}$-semimartingale has a $\mathcal{Q}$-compensator.

The weak-compactness assumption in Theorem 3.4 is optimal; see Theorem 5.4 below.

Corollary 3.5. Let $S$ be a $\mathbb{P}$-semimartingale. Then, it has a representation $S=$ $S_{0}+X+C$ with $X$ a $\mathcal{Q}$-supermartingale and $C$ a non decreasing process.

Proof: Take the canonical decomposition of $S$ in the form $S=S_{0}+S^{c}+V$, where $S_{0}$ is an $\mathcal{F}_{0}$-measurable random variable, $S^{c}$ is the continuous local martingale part of $S$, and $V$ is a process of finite variation on compact intervals. Let $B$ be a $\mathcal{Q}$-compensator of $S^{c}$. Then, $C=B+V^{+}$and $X=S-S_{0}-C$ prove the claim.

The next theorem is our second main result. Together with Theorem 3.4, they characterize the value process of optimal stopping under model ambiguity; see Remark 2.2, second part. The proof is presented in Section 7.

Theorem 3.6. Let $X$ be a $\mathbf{E}^{\downarrow}$-supermartingale. Then, under Assumption 3.1 there exists a probability measure $Q^{*} \in \mathcal{Q}$ under which $X$ is a local supermartingale. Hence, $X$ has a $\mathcal{Q}$-compensator.

We close this section with a few remarks. 
Remark 3.7. Let us give some background to Theorem 3.6's scope. Without the compactness assumption, there are examples of $\mathbf{E}^{\downarrow}$-supermartingales which are not semimartingales; see Treviño-Aguilar (2017, 2018). Theorem 5.4 below shows that a semimartingale does not necessarily have a $\mathcal{Q}$-compensator.

Remark 3.8. The Doob-Meyer decomposition theorem and the ODT can be seen related as briefly mentioned in the introduction. For example, in a Doob-Meyer decomposition under some conditions the martingale part can be written as a stochastic integral with respect to a semimartingale $W$ that has been apriori given. However there are important differences which we now discuss.

Generally speaking, in the Doob-Meyer decomposition the martingale part (or more specifically the $\mathcal{Q}$-supermartingale $X$ in Corollary 3.5) does not necessarily have such representation as stochastic integral, if not just because there is not an apriori given $W$, then because such representation does not exist (consider KunitaWatanabe decomposition where there is an orthogonal complement to a stochastic integral). In its formulation, the ODT applies only to $\mathcal{Q}$-supermartingales and $\mathcal{Q}$ is specifically a family of martingale measures for some apriori given semimartingale $W$. But an $\mathbf{E}^{\downarrow}$-supermartingale fails in general to be a $\mathcal{Q}$-supermartingale as we mentioned in Remark 3.7.

For the main motivation of the paper, model uncertainty, it makes sense to consider families $\mathcal{Q}$ which are not necessarily a family of martingale measures and they are within the scope of Corollary 3.5.

Remark 3.9. This is a follow up to Remark 2.2, second part. As we mentioned there, the value process of optimal stopping under model uncertainty denoted $U^{\downarrow}$ is an $\mathbf{E}^{\downarrow}$-supermartingale. The stopping time $\tau^{*}=\inf \left\{t \in[0, \mathbb{T}] \mid U_{t}^{\downarrow}=\mathrm{H}_{t}\right\}$ is optimal by Treviño-Aguilar (2017, Thm 2.1 and Lemma 2.1). Thus, the stochastic set $\left\{U^{\downarrow}=\right.$ $\mathrm{H}\}$ is crucial for $\tau^{*}$ and following classic terminology we call it the "stopping region". Under our Assumption 3.1 the process $U^{\downarrow}$ has a Doob-Meyer decomposition. Thus making it possible to study the stopping region with semimartingale techniques.

Remark 3.10. Specializing to $\mathbf{E}^{\downarrow}$-supermartingales arising as the value process in Remark 2.2, second part, we see that this value process can be written as $U^{\downarrow}=U_{0}^{\downarrow}+X+C$ where $X$ is a universal $\mathcal{Q}$-local-supermartingale and $C$ a non decreasing process. The family $\mathcal{Q}$ represents a set of priors in which a unique probability measure describing relevant distributions is uncertain and $\mathrm{H}$ is a reward process. An interpretation of $C$ is as the process accounting for model ambiguity. At first glance its non-decreasing paths might appear as unreasonable, since it suggests that as more information arrives ambiguity is not being reduced. However, this counterintuitive fact just reflects that risk and ambiguity are differentiated phenomena; see e.g., the discussion in Chen and Epstein (2002, p. 1406).

\section{Finite interaction}

It is to be expected that our concept of a $\mathcal{Q}$-compensator is closely related to Girsanov transformations of a semimartingale with respect to each member of the family $\mathcal{Q}$. In this section we formulate the concept of finite interaction in terms of compensators in Girsanov's transformations and establish equivalence with the property of having a $\mathcal{Q}$-compensator. The equivalent concept of finite interaction will prove to be more convenient in some specific situations as in the model developed in Section 5. 
We use the generic notation $Z$ for the density process with respect to $\mathbb{P}$ of a probability measure $Q$ in $\mathcal{Q}$. Thus, $Z_{t}=E\left[\frac{d Q}{d \mathbb{P}} \mid \mathcal{F}_{t}\right]$. We actually identify a probability measure $Q$ with $Z$ so we write $Z \in \mathcal{Q}$. The next definition is the main concept in this section.

Definition 4.1. Let $M$ be a continuous $\mathbb{P}$-local-martingale. We say $M$ has finite interaction with $\mathcal{Q}$ if there exists a non-decreasing process $B$ such that for all $Z \in \mathcal{Q}$

$$
\frac{1}{Z} \cdot\langle M, Z\rangle-B \text { is a non increasing process. }
$$

A semimartingale $S$ has finite interaction with $\mathcal{Q}$ if $S^{c}$ has finite interaction with $\mathcal{Q}$. If a semimartingale does not have finite interaction with $\mathcal{Q}$, then we say it has infinite interaction.

Theorem 4.2. Let $S$ be a $\mathbb{P}$-semimartingale having finite interaction with $\mathcal{Q}$. Then $S$ has a $\mathcal{Q}$-compensator.

Proof: Assume that $S$ has finite interaction with $\mathcal{Q}$. Under a Girsanov's transformation with respect to $Q \in \mathcal{Q}$, the semimartingale $S$ satisfies

$$
S=\bar{S}^{c}+\frac{1}{Z} \cdot\langle M, Z\rangle+V,
$$

where $\bar{S}^{c}=S^{c}-\frac{1}{Z} \cdot\langle M, Z\rangle$ is a $Q$-local-martingale; see Protter (2005, Theorem III.8.36 p. 133). There exists a non decreasing process $B$ such that $\frac{1}{Z} \cdot\langle M, Z\rangle$ is dominated by $B$ for all $Q \in \mathcal{Q}$ since $S^{c}$ has finite interaction with $\mathcal{Q}$. Then, $C=B+V^{+}$proves the claim.

The next result is the converse to Theorem 4.2.

Theorem 4.3. Let $S$ be a $\mathbb{P}$-semimartingale. If $S$ has a $\mathcal{Q}$-compensator then $S$ has finite interaction with $\mathcal{Q}$.

Proof: Let $C$ be a non decreasing process such that $X=S^{c}-C$ is a $\mathcal{Q}$-supermartingale. Under $Q \in \mathcal{Q}$ we have

$$
X=\bar{S}^{c}+\frac{1}{Z} \cdot\langle M, Z\rangle-C,
$$

where $\bar{S}^{c}$ is a $Q$-local-martingale. Now we will show that $\frac{1}{Z} \cdot\langle M, Z\rangle-C$ is a non increasing process. Let $X=M^{Q}-A^{Q}$ where $M^{Q}$ is a $Q$-local-martingale and $A^{Q}$ a non decreasing process. Then

$$
\bar{S}^{c}-M^{Q}=-A^{Q}-\frac{1}{Z} \cdot\langle M, Z\rangle+C .
$$

The left hand term is a $Q$-local-martingale while the right hand term is a process of finite variation. Hence the left hand is a purely discontinuous martingale (see Jacod and Shiryaev, 2003, Lemma I.4.14 (b)) and must be constant since it is continuous (see Jacod and Shiryaev, 2003, Lemma I.4.13 (b)). Thus, $\bar{S}^{c}=M^{Q}$ and $-A^{Q}=\frac{1}{Z} \cdot\langle M, Z\rangle-C$. Then, $\frac{1}{Z} \cdot\langle M, Z\rangle$ is dominated by $C$ in the order of non decreasing processes. Thus, $S^{c}$ has finite interaction with $\mathcal{Q}$. 


\section{A specific model}

In this section we illustrate the scope of our main results in Section 3 and show that the weak compactness assumption is optimal. To this end, we consider a specific model for a family of probability measures $\mathcal{Q}$ which is stable but initially not necessarily weakly compact. Note that in this section we work with the equivalent concept of finite interaction.

5.1. Definition of the family. The setting is as follows. We fix a continuous $d$ dimensional local martingale $M$ with the representation property described in Jacod and Shiryaev (2003, Definition III.4.22). Thus, if $N$ is a continuous local martingale, then there exists a predictable integrable integrand $\xi$ such that $N=\xi \cdot M$. Moreover, we fix a family of probability measures $\mathcal{Q}$ satisfying the conditions of the following assumption.

Assumption 5.1. Let $\mathcal{Q}$ be a convex family of probability measures equivalent to the probability measure $\mathbb{P}$ which we assume is also an element of $\mathcal{Q}$. Assume there exists a predictable mapping (a set valued function) $C: \Omega \times[0, \mathbb{T}] \rightrightarrows \mathbb{R}^{d}$ taking convex closed values such that the family of densities with respect to $\mathbb{P}$ of $\mathcal{Q}$ is given by

$$
\left\{\begin{array}{c}
q \text { predictable } \\
Z=\mathcal{E}\left(q \cdot M_{\mathbb{T}}\right) \mid \\
\quad Z \text { is a positive uniformly integrable martingale }
\end{array}\right\} .
$$

The family $\mathcal{Q}$ defined above is stable; see Delbaen (2006, Theorem 1).

5.1.1. Convex sets. Given that a main ingredient in the definition of the family $\mathcal{Q}$ is the convex valued mapping $C$, then, a few concepts from convex analysis are necessary.

If $C$ is a measurable mapping, recall that $\operatorname{dom}(C)$ is a subset of $\Omega \times[0, \mathbb{T}]$ where $C$ takes non-empty values.

We will assume a predictable "Motzkin decomposition" of the mapping $C$. It is given by $C=K+R$ where $K$ is a predictable compact- convex-valued mapping and $R$ is a predictable closed- convex- cone-valued mapping; see Goberna et al. (2010, Theorem 19) for characterizations of Motzkin decompositions. We will also assume that $0 \in K$.

5.2. Necessary and sufficient conditions for finite interaction. We need the next lemma for the proof of Theorem 5.4 below. The proof of the lemma is an easy exercise on measurable selections which by completeness is included.

Lemma 5.2. Let $q$ be a predictable process which is a selection of $C$. Then, there exists a predictable selection $a$ of $K$ and a predictable selection $b$ of $R$ such that $q=a+b$.

Proof: Take $q$ a predictable selection of $C$. Define a mapping by

$$
(\omega, t) \rightarrow\{(k, r) \in K(\omega, t) \times R(\omega, t) \mid k+r=q(\omega, t)\} .
$$

This is a predictable mapping due to Rockafellar and Wets (1998, Proposition 14.11). It is clearly closed-, convex-valued with full domain. By Rockafellar and Wets (1998, Corollary 14.6), there exists a predictable selection which gives the required decomposition of $q$. 
For $x, y \in \mathbb{R}^{d}$ let $(x, y)$ denote its usual inner product. The polar cone of a convex set $A$ is defined by

$$
A^{*}:=\left\{y \mid \sup _{x \in A}(x, y) \leq 0\right\} .
$$

From Theorem 3.4 we know that under the assumption of weak compactness, all semimartingales have a $\mathcal{Q}$-compensator and equivalently by Theorem 4.2 , that all semimartingales have finite interaction with $\mathcal{Q}$. In this section we do not assume weak compactness and give a very explicit characterization of a semimartingale having finite interaction with $\mathcal{Q}$.

We will represent the covariation matrix $\left(\left\langle M^{i}, M^{j}\right\rangle\right)_{i, j \leq d}$ as $m \cdot \mathbf{F}$ where $\mathbf{F}$ is a continuous non-decreasing locally-integrable predictable process and $m$ a predictable process taking symmetric and non-negative matrix values; see Jacod and Shiryaev (2003, Proposition II.2.9).

It is classical to define equivalent changes of probability measures by density processes $\left\{Z_{t}\right\}_{0 \leq t \leq \mathbb{T}}$ defined as Doléans-Dade exponentials. Always a critical step is to guarantee that the density process is a strictly positive and uniformly integrable martingale. The next lemma provides a manageable tool to generate a dense set of such densities. For two vectors $a, b \in \mathbb{R}^{d}$ we write $(a, m \cdot b)$ for the usual product $a^{t} m b$, where $a^{t}$ denotes the transpose of $a$.

Lemma 5.3. There exists a predictable $(0, \infty)$-valued process $\bar{b}$ such that for any predictable process $q$ with $(q, m \cdot q) \leq r \bar{b}$, for $r>0, q \cdot M$ is a martingale with bounded quadratic variation and the stochastic exponential $\mathcal{E}(\lambda q \cdot M)_{\mathbb{T}}$ defines an equivalent probability measure for any $\lambda>0$.

Proof: There exists a predictable $(0, \infty)$-valued process $\bar{b}$ with $(\bar{b} \cdot \mathbf{F})_{\mathbb{T}} \in L^{\infty}$ since $\mathbf{F}$ is locally integrable. Now $\mathcal{E}(\lambda q \cdot M)$ is a uniformly integrable martingale for any $\mathbb{R}^{d}$-valued predictable process $q$ with $(q, m \cdot q) \leq r \bar{b}$. Indeed, in this case $\lambda q \cdot M$ is a martingale of bounded quadratic variation and then a $B M O$-martingale; see Kazamaki (1994, Section 2.6). Thus, the stochastic exponential is a uniformly integrable martingale by Kazamaki (1994, Theorem 2.3 p. 31) and strictly positive by Kazamaki $(1994,(1.4))$.

The next theorem is the main result of this section. It determines the conditions under which a semimartingale has finite interaction with $\mathcal{Q}$ so that it has a $\mathcal{Q}$ compensator. Note that the Motzkin decomposition of the mapping $C$ is crucial and the result is quite intuitive. Note also that we recover the conclusion in Theorem 3.4 from this result. We introduce a control measure defined by $\mu(l):=E\left[\int 1_{l} d \mathbf{F}\right]$ for $l$ a predictable set.

Theorem 5.4. Let $S=S_{0}+N+V$ be a $\mathbb{P}$-semimartingale, where $V$ is a process of finite variation and $N$ a $\mathbb{P}$-local-martingale with representation $N=\eta \cdot M$. Then, $S$ has finite interaction with $\mathcal{Q}$ if and only if $\eta$ is $\mu$-a.s. valued in $(m R)^{*}$ and $B_{\mathbb{T}}<\infty$ $\mathbb{P}$-a.s. where $B$ is the predictable continuous non decreasing process

$$
B:=\sup _{r \in K}(\eta, m \cdot r)^{+} \cdot \mathbf{F} .
$$

Proof: Assume that $\eta$ is $\mu$-a.s. valued in $(m R)^{*}$ and $B_{\mathbb{T}}<\infty$. Under a probability measure $Q \in \mathcal{Q}$ with density process $Z$, the martingale $N$ takes the form

$$
\bar{N}+\frac{1}{Z} \cdot\langle N, Z\rangle,
$$


where $\bar{N}=N-\frac{1}{Z} \cdot\langle N, Z\rangle$ is a $Q$-local-martingale. For $Z=\mathcal{E}(q \cdot M)$ we have

$$
\frac{1}{Z} \cdot\langle N, Z\rangle=(\eta, m \cdot q) \cdot \mathbf{F}
$$

Represent $q=a+b$ where $a$ is $K$ valued and $b$ takes values in $R$; see Lemma 5.2. Then

$$
\frac{1}{Z} \cdot(N, Z) \leq(\eta, m \cdot a) \cdot \mathbf{F}, \mathbb{P}-a . s .
$$

Now

$$
\begin{aligned}
(\eta, m a) \cdot \mathbf{F}-B & =(\eta, m \cdot a)^{+} \cdot \mathbf{F}-(\eta, m \cdot a)^{-} \cdot \mathbf{F}-\left\{\sup _{r \in K}(\eta, m \cdot r)^{+}\right\} \cdot \mathbf{F} \\
& \leq\left\{(\eta, m \cdot a)^{+}-\sup _{r \in K}(\eta, m \cdot r)^{+}\right\} \cdot \mathbf{F}
\end{aligned}
$$

with the right hand side clearly non-increasing.

Conversely, assume that $l=\left\{\eta \notin(m R)^{*}\right\}$ is such that $\mu(l)>0$. Let $J=\{b \in$ $R \mid(\eta, m \cdot b)>0$ and $(b, m \cdot b) \leq \bar{b}\}$ where $\bar{b}$ is the predictable process of Lemma 5.3. Note that $\operatorname{dom}(J)=l$ since $R$ is cone valued. Let $J_{\epsilon}=R \cap\left\{b \in \mathbb{R}^{d} \mid(\eta, m \cdot b) \geq\right.$ $\epsilon\} \cap\left\{b \in \mathbb{R}^{d} \mid(b, m \cdot b) \leq \bar{b}\right\}$. Then $J_{\epsilon}$ is predictable and decreases for the parameter $\epsilon$. Moreover, $J=\bigcup_{\epsilon>0} J_{\epsilon}$. Lets check the predictability of $J_{\epsilon}$. Let $M: \Omega \times \mathfrak{m} \rightrightarrows \mathbb{R}$ be defined by $M(a):=\{b \mid(b, a \cdot b) \leq 1\}$ where $\mathfrak{m}$ denotes the family of square matrices of dimension $d$. Then $M\left(\bar{b}^{-1} m\right)$ is predictable by Rockafellar and Wets (1998, Corollary 14.14). Thus, the mapping $\left\{b \in \mathbb{R}^{d} \mid(b, m \cdot b) \leq \bar{b}\right\}$ is predictable.

The mapping $A_{1}:=\left\{b \in \mathbb{R}^{d} \mid(\eta \cdot m, b) \leq 1\right\}$ is predictable by Rockafellar and Wets $\left(1998\right.$, Exercise 14.12 e)) so $A_{\epsilon}:=\epsilon A_{1}$ is predictable by Rockafellar and Wets (1998, Theorem 14.13). Then, $\left\{b \in \mathbb{R}^{d} \mid(\eta, m \cdot b) \geq \epsilon\right\}$ is predictable since it is the closure of the complement of $A_{\epsilon}$.

We have just checked that $J_{\epsilon}$ is predictable. Choose $J_{\epsilon}$ with $\mu\left(J_{\epsilon}\right)>0$. There is a selection of $J_{\epsilon}$ on $\operatorname{dom}\left(J_{\epsilon}\right)$ by Rockafellar and Wets (1998, Corollary 14.6). Let $b$ be equal to a selection of $J_{\epsilon}$ on $\operatorname{dom}\left(J_{\epsilon}\right)$ and zero otherwise. For $\lambda>0$, let $q^{\lambda}=\lambda b$ and $Z^{\lambda}=\mathcal{E}\left(q^{\lambda} \cdot M\right)$. Note that $Z^{\lambda}$ defines an element of $\mathcal{Q}$ by Lemma 5.3. Then, with positive probability

$$
\lim _{\lambda \rightarrow \infty} \frac{1}{Z^{\lambda}} \cdot\left\langle N, Z^{\lambda}\right\rangle_{\mathbb{T}}=\lim _{\lambda \rightarrow \infty} \lambda(\eta, m \cdot b) \cdot \mathbf{F}_{\mathbb{T}}=\infty .
$$

Thus, $S$ has infinite interaction with $\mathcal{Q}$.

Now assume $\mu(l)=0$ but $B_{\mathbb{T}}=\infty$ with positive probability. By Aliprantis and Border (2006, Theorem 18.19) there exists a predictable process $a^{*}$ selection of $K$ with

$$
\left(\eta(\omega, t), m(\omega, t) \cdot a^{*}(\omega, t)\right)^{+}=\sup _{r \in K(\omega, t)}(\eta(\omega, t), m(\omega, t) \cdot r)^{+}
$$

Note that we can choose $a^{*}$ in such a way that

$$
\left(\eta(\omega, t), m(\omega, t) \cdot a^{*}(\omega, t)\right)^{+}=\left(\eta(\omega, t), m(\omega, t) \cdot a^{*}(\omega, t)\right)
$$

since $0 \in K$. Let $\epsilon_{\lambda}^{2}:=1 \wedge \frac{\lambda \bar{b}}{\left(a^{*}, m \cdot a^{*}\right)}$ for $\lambda>0$. Then,

$$
a_{\lambda}:=a^{*} \epsilon_{\lambda}
$$

is a predictable $K$-valued process and $\left(a_{\lambda}, m \cdot a_{\lambda}\right) \leq \lambda \bar{b}$, hence $Z_{\lambda}:=\mathcal{E}\left(a_{\lambda} \cdot M\right)$ defines an element of $\mathcal{Q}$. Now

$$
\frac{1}{Z_{\lambda}} \cdot\left\langle N, Z_{\lambda}\right\rangle=\left(\eta, m \cdot a_{\lambda}\right)^{+} \cdot \mathbf{F} .
$$


We have that $\lim _{\lambda \rightarrow \infty}\left(\eta, m \cdot a_{\lambda}\right)^{+}=\left(\eta, m \cdot a^{*}\right)^{+}$pointwise monotonically. In particular, $\lim _{\lambda \rightarrow \infty}\left(\eta, m \cdot a_{\lambda}\right)^{+} \cdot \mathbf{F}_{\mathbb{T}}=\infty$ with positive probability. Hence, there is no finite non-decreasing process $C$ dominating $\left\{\frac{1}{Z_{\lambda}} \cdot\left\langle N, Z_{\lambda}\right\rangle\right\}_{\lambda>0}$. Thus, $S$ has again infinite interaction with $\mathcal{Q}$.

Remark 5.5. Consider the case in which $\mathcal{Q}$ is the set of equivalent local martingale measures of a process $S$. Let $X$ be a $\mathcal{Q}$-supermartingale with $\mathbb{P}$-canonical decomposition $X=X_{0}+M+V$. Now consider the Kunita-Watanabe decomposition of $M$ to give $X=X_{0}+\xi \cdot S+N+V$, where $N$ is orthogonal to $S$. Well-known arguments show that $N$ must be zero. So $X=X_{0}+\int \xi d S+V$. Take a density $Z$ of an element of $\mathcal{Q}$ that can be written as $Z=\mathcal{E}(L)$ where $L$ is a $\mathbb{P}$-local-martingale orthogonal to $S$. Then

$$
\frac{1}{Z} \cdot\langle\xi \cdot S, Z\rangle=\xi \cdot\langle S, L\rangle=0 .
$$

Thus, we see that a $\mathcal{Q}$-supermartingale has finite interaction with elements of $\mathcal{Q}$ represented as stochastic exponentials.

The next corollary confirms Theorem 3.4 together with its Corollary 3.5. Taking into account the more explicit structure of $\mathcal{Q}$ in this section, it also identifies compensators.

Corollary 5.6. If $\mathcal{Q}$ is weakly compact, then all $\mathbb{P}$-semimartingales have finite interaction with $\mathcal{Q}$. Moreover, for each local martingale $N$ there exists a probability measure $Q^{*}$ under which $N$ is a local submartingale and a $\mathcal{Q}$-compensator is given by (5.2) which is the finite variation process in the canonical decomposition with respect to $Q^{*}$.

Proof: Our fist task is to verify that under the hypotheses of the corollary, the conditions in Theorem 5.4 are satisfied for any semimartingale.

Under the conditions of the corollary, any predictable $M$-integrable process $\eta$ must be $(m R)^{*}$-valued, $\mu$-a.s. In order to prove this property, we claim that

$$
\mu\left(\operatorname{dom}\left(C_{0}\right)\right)=0, \text { where } C_{0}:=\{b \in R \mid(b, m \cdot b)>0\} .
$$

Lets assume the claim (5.5) and show the required property. Note that (5.5) yields $(b, m \cdot b)=0, \mu$-a.s. for any $R$-valued predictable process $b$. For any such process, its integral $b \cdot M$ is constant and equal to zero since $\langle b \cdot M\rangle=(b, m \cdot b) \cdot \mathbf{F}=0$, $\mathbb{P}$-a.s. Moreover, $(\eta, m \cdot b) \cdot \mathbf{F}=\langle\eta \cdot M, b \cdot M\rangle=0$, showing that $\eta$ is $(m R)^{*}$-valued, $\mu$-a.s. Now we verify the claim (5.5). Let $\bar{b}$ be as fixed in the proof of Lemma 5.3. Let $C_{1}=\{b \in R \mid(b, m \cdot b)=\bar{b}\}$. Note that $\operatorname{dom}\left(C_{1}\right)=\operatorname{dom}\left(C_{0}\right)$ since $R$ is a cone and $\bar{b}$ is strictly positive. Take a predictable selection $b_{0}$ of $C_{1}$ on $\operatorname{dom}\left(C_{1}\right)$ and extend it otherwise identically equal to zero. Then, $b_{0} \cdot M$ has bounded quadratic variation and for $\lambda>0$ the density $\mathcal{E}\left(\lambda b_{0} \cdot M\right)$ defines an element of $\mathcal{Q}$. It converges pointwise to a random variable $Z^{*}$ which is equal to zero on the set $\left\{\left\langle b_{0} \cdot M\right\rangle_{\mathbb{T}}>0\right\}$. By weak-compactness, $Z^{*}$ must be an element of $\mathcal{Q}$ so $\left\{\left\langle b_{0} \cdot M\right\rangle_{\mathbb{T}}>0\right\}$ must be a $\mathbb{P}$-null event. Hence $\mu\left(\operatorname{dom}\left(C_{1}\right)\right)=0$ as claimed.

Now we prove that $B_{\mathbb{T}}<\infty$ where $B$ is the process in (5.2). Let $a^{*}$ be a predictable selection of $K$ satisfying (5.3). Let $a_{j}$ be defined by $a_{j}=1_{D_{j}} a^{*}$ where for $j \in \mathbf{N}, D_{j}$ is the predictable set defined by $D_{j}=\left\{\left(a^{*}, m \cdot a^{*}\right) \leq j \bar{b}\right\}$. The densities $Z_{j}=\mathcal{E}\left(a_{j} \cdot M\right)$ define a sequence in $\mathcal{Q}$. By monotone convergence $a_{j} \cdot\langle M\rangle_{\mathbb{T}}$ converges to $a^{*} \cdot\langle M\rangle_{\mathbb{T}} \mathbb{P}$-a.s. Let $\mathbf{V}$ be the process defined in Lemma 6.2 for the 
family $\mathcal{Q}$. Note that $a_{j} \cdot\langle M\rangle_{\mathbb{T}} \leq \mathbf{V}_{\mathbb{T}}$ implying that $a^{*} \cdot\langle M\rangle_{\mathbb{T}}<\infty$. Thus, $a^{*} \cdot M$ is locally a square integrable martingale. Now $\left(a_{j} \cdot M\right)_{\mathbb{T}}$ converges $\mathbb{P}$-a.s. along a subsequence to $\left(a^{*} \cdot M\right)_{\mathbb{T}}$ due to Jacod and Shiryaev (2003, Theorem I.4.40). As a consequence, $Z^{*}:=\mathcal{E}\left(a^{*} \cdot M\right) \in \mathcal{Q}$. We have that $\inf _{s} Z_{s}^{*}>0$ and $\left\langle Z^{*}, \eta \cdot M\right\rangle$ is a process of finite variation by Jacod and Shiryaev (2003, Theorem I.4.2 p.38). Thus $\frac{1}{Z^{*}} \cdot\left\langle Z^{*}, \eta \cdot M\right\rangle$ is a process of finite variation which is equal to $B$ due to (5.3). Hence $B_{\mathbb{T}}<\infty, \mathbb{P}$-a.s.

For the last claim take the probability measure $Q^{*}$ determined by $Z^{*}$ and consider the Girsanov's transformation of $N$ with respect to $Q^{*}$.

5.3. A counterexample. In Remark 3.7 we claimed the existence of stable families of probability measures $\mathcal{Q}$ such that there are $\mathbb{P}$-semimartingales without a $\mathcal{Q}$ compensator. In this subsection we give a specific example of this fact that in particular show that the weak-compactness assumption in Theorem 3.4 is crucial and cannot be dropped out.

Let $M$ be a continuous $\mathbb{P}$-local-martingale with $M_{0}=0$ and with the representation property described in Jacod and Shiryaev (2003, Definition III.4.22). Let $K(\omega, t)=\{0\}, R(\omega, t)=\mathbf{R}_{+}$and $C(\omega, t)=\mathbf{R}_{+}$for $(\omega, t) \in \Omega \times[0, \mathbb{T}]$. With this specification we define a family $\mathcal{Q}$ of probability measures as in Subsection 5.1. Let $S=M$ so $S_{t}=\int_{0}^{t} \eta_{t} d M_{t}$ with $\eta \equiv 1$. It is clear that $S$ is a $\mathbb{P}$-semimartingale. Moreover, $S$ has infinite interaction with $\mathcal{Q}$. Indeed, by Theorem 5.4 there are two conditions that $S$ has to satisfy and the first clearly fails $\left(\eta\right.$ must be $(m R)^{*}$ valued $\mu$-a.s.) since for our specific choice $\eta \equiv 1$ and $(m R)^{*}=\left(\mathbf{R}_{+}\right)^{*}=\mathbf{R}_{-}$. As a consequence, $S$ does not have a $\mathcal{Q}$-compensator due to Theorem 4.3.

\section{Proof of Theorem 3.4}

In this section, let $\mathcal{Q}$ be a family of probability measures satisfying Assumption 3.1. Let $\mathcal{N}$ be the class of all local martingales $N$ such that $\mathcal{E}(N)$ is the density process of a probability measure in $\mathcal{Q}$. The family $\mathcal{Q}$ then can always be represented as $\mathcal{Q}=\{\mathcal{E}(N) \mid N \in \mathcal{N}\}$; see Remark 3.2.

The next lemma shows that the Assumption 3.1 yields a property for the class $\mathcal{N}$ known as predictable convexity; see Föllmer and Kramkov (1997).

Lemma 6.1. The class $\mathcal{N}$ is predictably convex: For any predictable process $h$ taking values in the interval $[0,1]$ and $N^{1}, N^{2} \in \mathcal{N}$ we have $N=h \cdot N^{1}+(1-h) \cdot N^{2} \in$ $\mathcal{N}$.

Proof: We denote by $\mathscr{A}$ the class of predictable sets generated by finite unions of the stochastic intervals $\llbracket 0_{A} \rrbracket$ and $\left(T_{1}, T_{2} \rrbracket\right.$ for $A \in \mathcal{F}_{0}$ and $T_{1}, T_{2}$ stopping times. For two sets $A$ and $B$ let $A \Delta B$ denote their symmetric difference. We start with the case $h=1_{A}$ where $A$ is a predictable set. The proof is based on Delbaen (2006, Lemmas 2 and 3) but there are some additional details to be solved. Let $\mu$ be a predictable measure such that $d \mathbb{P} \otimes d\left\langle N^{1}\right\rangle$ and $d \mathbb{P} \otimes d\left\langle N^{2}\right\rangle$ are absolutely continuous with respect to $\mu$. Let $A_{k}$ be a sequence of predictable sets in $\mathscr{A}$ such that $\mu\left(A \Delta A_{k}\right)$ converges to zero. It is clear from the stability of $\mathcal{Q}$ that $N^{k} \in \mathcal{N}$ for

$$
N^{k}=1_{A_{k}} \cdot N^{1}+1_{A_{k}^{c}} \cdot N^{2} .
$$


Moreover, $N_{\mathbb{T}}^{k}$, respectively $\left\langle N^{k}\right\rangle_{\mathbb{T}}$, converge in probability to $N_{\mathbb{T}}$, respectively $\langle N\rangle_{\mathbb{T}}$; this can be shown by adapting the proof of Jacod and Shiryaev (2003, Proposition III.6.9) as follows. Let $M^{k}=N-N^{k}$ so

$\left\langle M^{k}\right\rangle=\left(1_{A}-1_{A_{k}}\right)^{2} \cdot\left\langle N^{1}\right\rangle+2\left(1_{A}-1_{A_{k}}\right)\left(1_{A^{c}}-1_{A_{k}^{c}}\right) \cdot\left\langle N^{1}, N^{2}\right\rangle+\left(1_{A^{c}}-1_{A_{k}^{c}}\right)^{2} \cdot\left\langle N^{2}\right\rangle$.

Hence $\left\langle M^{k}\right\rangle_{\mathbb{T}}$ converges in probability to zero and along a subsequence, which we do not relabel, the convergence is $\mathbb{P}$-a.s. Then, for a localizing sequence $\left\{T_{n}\right\}_{n \in \mathbf{N}}$ of $N^{1}$ and $N^{2}$ we have

$$
\lim _{k \rightarrow \infty} E\left[\left\langle M_{k}\right\rangle_{T_{n}}\right]=0 .
$$

Now $\mathcal{E}_{\mathbb{T}}\left(N^{T_{n}}\right)$ is the pointwise limit of $\mathcal{E}_{\mathbb{T}}\left(\left(N^{k}\right)^{T_{n}}\right)$. From this, we easily obtain that $\mathcal{E}_{\mathbb{T}}(N)$ determines a probability measure in $\mathcal{Q}$ by weak compactness and $N \in \mathcal{N}$. The general case of a predictable $h$ follows now as in Delbaen (2006, Lemma 11).

Lemma 6.2. Let

$$
\mathbf{V}:=\operatorname{ess} \sup \{\langle N\rangle \mid N \in \mathcal{N}\} .
$$

Then, $\mathbf{V}$ is a continuous non-decreasing process with $\mathbf{V}_{\mathbb{T}}<\infty \mathbb{P}$-a.s.

Proof: For the definition of $\mathbf{V}$ we first precise the meaning of ess sup in the sense of Dellacherie (1978). By Dellacherie (1978, Theorem 2) there exists a sequence $\left\{N^{i}\right\}_{i \in \mathbf{N}} \subset \mathcal{N}$ such that $\mathbf{V}=\sup _{i}\left\langle N^{i}\right\rangle$ and by the predictable convexity of $\mathcal{N}$ proved in Lemma 6.1 this sequence can actually be taken monotone increasing in the order of non decreasing processes. Note that this already implies that $\mathbf{V}$ is a non-decreasing process. For $\mathcal{A}:=\left\{\mathbf{V}_{\mathbb{T}}=\infty\right\}$, we must show $\mathbb{P}(\mathcal{A})=0$. To this end, we claim that it is possible to derive from $\left\{N^{i}\right\}_{i \in \mathbf{N}}$ a further sequence $\left\{\tilde{N}^{k}\right\}_{k \in \mathbf{N}} \subset \mathcal{N}$ with the property

$$
\mathcal{A} \subseteq\left\{\lim _{k \rightarrow \infty}\left(\tilde{N}_{\mathbb{T}}^{k}-\frac{1}{2}\left\langle\tilde{N}^{k}\right\rangle_{\mathbb{T}}\right)=-\infty\right\} .
$$

In this case we obtain for $Z^{k}=\mathcal{E}_{\mathbb{T}}\left(\tilde{N}^{k}\right)$ that

$$
\lim _{k \rightarrow \infty} E\left[Z^{k} 1_{\mathcal{A}}\right]=E\left[\lim _{k \rightarrow \infty} Z^{k} 1_{\mathcal{A}}\right]=0,
$$

where the first equality is due to the uniform integrability and the second by (6.2) and the definition of $Z^{k}$. Let $Z^{*}$ be the weak limit of $Z^{k}$ along a subsequence, so $Z^{*} \in \mathcal{Q}$. Then

$$
E\left[Z^{*} 1_{\mathcal{A}}\right]=\lim _{k \rightarrow \infty} E\left[Z^{k} 1_{\mathcal{A}}\right]
$$

implying that $\mathbb{P}(\mathcal{A})=0$. In order to show (6.2) we go through several steps.

(1) Let

$$
T_{i, j}:=\inf \left\{t \in[0, \mathbb{T}] \mid\left\langle N^{i}\right\rangle_{t} \geq j\right\}, \inf \emptyset=\infty .
$$

We clearly have that

$$
\mathcal{A}=\bigcap_{j} \bigcup_{i}\left\{T_{i, j}<\infty\right\}
$$


An equality from which we derive the existence of increasing subsequences $\left\{i_{k}\right\}_{k \in \mathbf{N}}$ and $\left\{j_{k}\right\}_{k \in \mathbf{N}}$ with

$$
\begin{array}{r}
\sum_{k=1}^{\infty} j_{k}^{-\frac{1}{3}}<\infty \\
\sum_{k=1}^{\infty} \mathbb{P}\left(\mathcal{A} \Delta\left\{T_{i_{k}, j_{k}}<\infty\right\}\right)<\infty .
\end{array}
$$

We sketch how to verify (6.4). Note that $\mathcal{A}=\bigcap_{j \in \mathbf{N}}\left\{\mathbf{V}_{\mathbb{T}} \geq j\right\}$ so

$$
\lim _{j \rightarrow \infty} \mathbb{P}\left(\left\{\mathbf{V}_{\mathbb{T}} \geq j\right\} \backslash \mathcal{A}\right)=0 .
$$

Also, $\left\{\mathbf{V}_{\mathbb{T}} \geq j\right\}=\bigcup_{i \in \mathbf{N}}\left\{T_{i, j}<\infty\right\}$ so

$$
\lim _{i \rightarrow \infty} \mathbb{P}\left(\left\{\mathbf{V}_{\mathbb{T}} \geq j\right\} \backslash\left\{T_{i, j}<\infty\right\}\right)=0
$$

For $\epsilon>0$ let $j(\epsilon)$ be such that

$$
\mathbb{P}\left(\left\{\mathbf{V}_{\mathbb{T}} \geq j(\epsilon)\right\} \backslash \mathcal{A}\right) \leq \epsilon / 2,
$$

once $j(\epsilon)$ has been fixed, let $i(\epsilon)$ be such that

$$
\mathbb{P}\left(\left\{\mathbf{V}_{\mathbb{T}} \geq j(\epsilon)\right\} \backslash\left\{T_{i(\epsilon), j(\epsilon)}<\infty\right\}\right) \leq \epsilon / 2 .
$$

The obvious inclusion

$$
\mathcal{A} \backslash\left\{T_{i(\epsilon), j(\epsilon)}<\infty\right\} \subset\left\{\mathbf{V}_{\mathbb{T}} \geq j(\epsilon)\right\} \backslash\left\{T_{i(\epsilon), j(\epsilon)}<\infty\right\}
$$

yields $\mathbb{P}\left(\mathcal{A} \backslash\left\{T_{i(\epsilon), j(\epsilon)}<\infty\right\}\right) \leq \epsilon / 2$. The inclusion

$$
\left\{T_{i(\epsilon), j(\epsilon)}<\infty\right\} \backslash \mathcal{A} \subset\left\{\mathbf{V}_{\mathbb{T}} \geq j(\epsilon)\right\} \backslash \mathcal{A}
$$

yields $\mathbb{P}\left(\left\{T_{i(\epsilon), j(\epsilon)}<\infty\right\} \backslash \mathcal{A}\right) \leq \epsilon / 2$. To conclude the verification of (6.4) select a summable sequence of positive $\epsilon$ 's. To obtain simultaneously (6.3) and (6.4) we choose conveniently a sequence satisfying (6.3) and then extract further subsequences through a diagonalization argument.

(2) Take increasing sequences $\left\{i_{k}\right\}_{k \in \mathbf{N}}$ and $\left\{j_{k}\right\}_{k \in \mathbf{N}}$ satisfying (6.3) and (6.4). Let $\tilde{N}^{k}$ be defined by $\tilde{N}_{t}^{k}=N_{t \wedge T_{i_{k}, j_{k}}}^{i_{k}}$. We claim

$$
\mathbf{V}_{\mathbb{T}}=\lim _{k \rightarrow \infty}\left\langle\tilde{N}^{k}\right\rangle_{\mathbb{T}}
$$

Indeed, for all $k^{\prime}>k$ we have $\left\{\mathbf{V}_{\mathbb{T}}<j_{k}\right\} \subset\left\{T_{i_{k^{\prime}}, j_{k^{\prime}}}=\infty\right\}$. Hence, on $\left\{\mathbf{V}_{\mathbb{T}}<j_{k}\right\}$ we have $\left\langle\tilde{N}^{k^{\prime}}\right\rangle_{\mathbb{T}}=\left\langle N^{i_{k^{\prime}}}\right\rangle_{\mathbb{T}}$ showing the claim on $\mathcal{A}^{c}$. The equation (6.4) yields that the set

$$
\limsup _{k \rightarrow \infty}\left[\mathcal{A} \Delta\left\{T_{i_{k}, j_{k}}<\infty\right\}\right]
$$

is $\mathbb{P}$-null due to Borel-Cantelli lemma. Then, the following set is also a null set

$$
\limsup _{k \rightarrow \infty}\left[\mathcal{A} \cap\left\{T_{i_{k}, j_{k}}=\infty\right\}\right]
$$

This means that outside a null set, for $\omega \in \mathcal{A}$ exists $k_{0}(\omega)$ such that for $k \geq k_{0}(\omega)$ it follows that $T_{i_{k}, j_{k}}(\omega)<\infty$. Hence, $N_{T_{i_{k}, j_{k}}}^{i_{k}}$ evaluated in $\omega$ is equal to $j_{k}$ which clearly diverges to $\infty$. This shows the claim on $\mathcal{A}$ for $k \rightarrow \infty$. 
(3) Without loss of generality assume $E\left[\left\langle\tilde{N}^{1}\right\rangle_{\mathbb{T}}^{\frac{1}{2}}\right]>0$. Let $\lambda^{k}=$ $j_{k}^{1 / 3} C E\left[\left\langle\tilde{N}^{k}\right\rangle_{\mathbb{T}}^{\frac{1}{2}}\right]$ where $C$ is the constant in Burkholder-Davis-Gundy inequalities. Then

$$
\mathbb{P}\left(\left|\tilde{N}_{\mathbb{T}}^{k}\right| \geq \lambda^{k}\right) \leq \frac{1}{\lambda^{k}} E\left[\left|\tilde{N}_{\mathbb{T}}^{k}\right|\right] \leq \frac{1}{\lambda^{k}} C E\left[\left\langle\tilde{N}^{k}\right\rangle_{\mathbb{T}}^{\frac{1}{2}}\right]=j_{k}^{-\frac{1}{3}}
$$

where we obtain the first inequality from Doob's maximal inequality and the second follows from Burkholder-Davis-Gundy inequalities. Note that

$$
\begin{aligned}
& \mathcal{A} \backslash \liminf _{k \rightarrow \infty}\left[\left\{\left|\tilde{N}_{\mathbb{T}}^{k}\right|<\lambda^{k}\right\} \cap\left\{T_{i_{k}, j_{k}}<\infty\right\}\right] \\
= & \mathcal{A} \cap\left(\liminf _{k \rightarrow \infty}\left[\left\{\left|\tilde{N}_{\mathbb{T}}^{k}\right|<\lambda^{k}\right\} \cap\left\{T_{i_{k}, j_{k}}<\infty\right\}\right]\right)^{c} \\
= & \mathcal{A} \cap \limsup _{k \rightarrow \infty}\left[\left\{\left|\tilde{N}_{\mathbb{T}}^{k}\right|<\lambda^{k}\right\}^{c} \cup\left\{T_{i_{k}, j_{k}}<\infty\right\}^{c}\right] \\
= & \limsup \left[\left(\mathcal{A} \cap\left\{\left|\tilde{N}_{\mathbb{T}}^{k}\right| \geq \lambda^{k}\right\}\right) \cup\left(\mathcal{A} \cap\left\{T_{i_{k}, j_{k}}=\infty\right\}\right)\right] .
\end{aligned}
$$

The equations (6.3) and (6.4) together with (6.5) yield that lim sup in the last equality is $\mathbb{P}$-null. Hence

$$
\mathcal{A} \subseteq \liminf _{k \rightarrow \infty}\left[\left\{\left|\tilde{N}_{\mathbb{T}}^{k}\right|<\lambda^{k}\right\} \cap\left\{T_{i_{k}, j_{k}}<\infty\right\}\right], \mathbb{P}-\text { a.s. }
$$

(4) Now (6.2) will follow after we verify that

$$
\liminf _{k \rightarrow \infty}\left[\left\{\left|\tilde{N}_{\mathbb{T}}^{k}\right|<\lambda^{k}\right\} \cap\left\{T_{i_{k}, j_{k}}<\infty\right\}\right] \subseteq\left\{\lim _{k \rightarrow \infty}\left(\tilde{N}_{\mathbb{T}}^{k}-\frac{1}{2}\left\langle\tilde{N}^{k}\right\rangle_{\mathbb{T}}\right)=-\infty\right\} .
$$

We estimate

$$
\tilde{N}_{\mathbb{T}}^{k}-\frac{1}{2}\left\langle\tilde{N}^{k}\right\rangle_{\mathbb{T}}=\left(\tilde{N}_{\mathbb{T}}^{k}-\lambda^{k}\right)+\left(\lambda^{k}-\frac{1}{2}\left\langle\tilde{N}^{k}\right\rangle_{\mathbb{T}}\right)
$$

The first term on the right hand side is non positive on $\left\{\left|\tilde{N}_{\mathbb{T}}^{k}\right|<\lambda^{k}\right\}$ (which has probability close to one due to (6.5)). For the second, note first that $\lambda^{k} \leq C j_{k}^{\frac{5}{6}}$ and on the event $\left\{T_{i_{k}, j_{k}}<\infty\right\}$ we have $\left\langle\tilde{N}^{k}\right\rangle_{\mathbb{T}}=j_{k}$ and

$$
\lambda^{k}-\frac{1}{2}\left\langle\tilde{N}^{k}\right\rangle_{\mathbb{T}} \leq C j_{k}^{\frac{5}{6}}-\frac{1}{2} j_{k} .
$$

Hence, on the event $\left\{\left|\tilde{N}_{\mathbb{T}}^{k}\right|<\lambda^{k}\right\} \cap\left\{T_{i_{k}, j_{k}}<\infty\right\}$ we have

$$
\tilde{N}_{\mathbb{T}}^{k}-\frac{1}{2}\left\langle\tilde{N}^{k}\right\rangle_{\mathbb{T}} \leq C j_{k}^{\frac{5}{6}}-\frac{1}{2} j_{k}
$$

The previous inequality implies the required inclusion in this step since $\lim _{k \rightarrow \infty}\left(C j_{k}^{\frac{5}{6}}-\frac{1}{2} j_{k}\right)=-\infty$

(5) It only remains to show that $\mathbf{V}$ is a continuous process. This follows from the fact that the paths of $\left\langle N^{i}\right\rangle$ converge uniformly to the paths of $\mathbf{V}$ on $\mathcal{A}^{c}$. 
The next lemma concludes the proof of Theorem 3.4. For a process of finite variation $V$ we use the notation $V_{t v}$ for its total variation process: $V_{t v}=V^{+}+V^{-}$.

Lemma 6.3. Let $L$ be a local martingale. Then

$$
B=\operatorname{ess} \sup \{\langle L, N\rangle \mid N \in \mathcal{N}\}
$$

is a non-decreasing process, $B_{\mathbb{T}}<\infty$, and is a $\mathcal{Q}$-compensator of $L$.

Proof: One easily sees from Lemma 6.1 that the family $\{\langle L, N\rangle \mid N \in \mathcal{N}\}$ is predictably convex. Moreover, for any $N \in \mathcal{N}$ the process $\langle L, N\rangle^{+}$is an element of the family. Indeed, there exists a predictable set $p \subset \Omega \times[0, \mathbb{T}]$ such that $\langle L, N\rangle^{+}=1_{p} \cdot\langle L, N\rangle$ and certainly $1_{p} \cdot N \in \mathcal{N}$. Thus, in the essential supremum of (6.7) one can restrict to elements $N$ with a non-decreasing bracket $\langle L, N\rangle$.

By Kunita-Watanabe inequality, see e.g., Protter (2005, Theorem II.25 p.69), we have

$$
\begin{aligned}
\langle L, N\rangle_{t v} & \leq \sqrt{\langle L\rangle} \sqrt{\langle N\rangle} \\
& \leq \sqrt{\langle L\rangle} \sqrt{\mathbf{V}}
\end{aligned}
$$

where $\mathbf{V}$ is the process defined in Lemma 6.2. As a consequence, the process (6.7) is non-decreasing continuous and satisfies $B_{\mathbb{T}}<\infty$, as claimed.

For $N \in \mathcal{N}$ consider the Girsanov transformation of $L$ with respect to $Z=\mathcal{E}(N)$

$$
L=\bar{L}+\frac{1}{Z} \cdot\langle L, Z\rangle=\bar{L}+\langle L, N\rangle
$$

where $\bar{L}=L-\frac{1}{Z} \cdot\langle L, Z\rangle$ is a $Q$-local-martingale. By construction $\langle L, N\rangle-B$ is a non-increasing process. Thus, $B$ is indeed a $\mathcal{Q}$-compensator of $L$.

\section{Proof of Theorem 3.6}

Proof: We will take $\mathbb{T}=1$. Assume first that $X$ is bounded. Let $D_{n}=\left\{\frac{i}{2^{n}} \mid\right.$ $\left.i=0 \ldots 2^{n}\right\}$ and $D=\bigcup_{n=1}^{\infty} D_{n}$. The restriction of $X$ to each $D_{n}$ is a $\mathbf{E}^{\downarrow}$ supermartingale. Then, there exists a probability measure $Q_{n} \in \mathcal{Q}$ such that $X$ is a $Q_{n}$-supermartingale on $D_{n}$, due to Riedel (2009, Lemma 6). Thus, for $t, s \in D_{n}$ with $t>s$ we have

$$
E_{Q_{n}}\left[X_{t} \mid \mathcal{F}_{s}\right] \leq X_{s}
$$

Let $\left\{Q_{n}\right\}_{n \in \mathbb{N}}$ be a sequence of probability measures constructed in this way considering the sequence of dyadic numbers $\left\{D_{n}\right\}_{n \in \mathbb{N}}$. By taking a subsequence if necessary, assume the sequence of densities converges weakly to a density defining a probability measure $Q^{*} \in \mathcal{Q}$. Lets see that $X$ is a $Q^{*}$-supermartingale when indexed by times in $D$. For $t, s \in D_{n_{0}}$ with $t>s$ and $A \in \mathcal{F}_{s}$ we have

$$
E_{Q^{*}}\left[X_{t} 1_{A}\right]=\lim _{n \rightarrow \infty} E_{Q_{n}}\left[X_{t} 1_{A}\right] \leq \lim _{n \rightarrow \infty} E_{Q_{n}}\left[X_{s} 1_{A}\right]=E_{Q^{*}}\left[X_{s} 1_{A}\right]
$$

Hence, we have $E_{Q^{*}}\left[X_{t} \mid \mathcal{F}_{s}\right] \leq X_{s}$, showing the claim for times in $D$. Now the $\mathbf{E}^{\downarrow}$-supermartingale property transfers to the interval $[0, \mathbb{T}]$ by the right continuity of $X$. Take $t \in[0, \mathbb{T}), s \in D$ with $s<t$ and $A \in \mathcal{F}_{s}$. For $\left\{t_{n}\right\}_{n \in \mathbb{N}} \subset D$ decreasing 
to $t$, we have

$$
\begin{aligned}
E_{Q^{*}}\left[\limsup _{n \rightarrow \infty} E_{Q^{*}}\left[X_{t_{n}} 1_{A} \mid \mathcal{F}_{s}\right]\right] & \geq \limsup _{n \rightarrow \infty} E_{Q^{*}}\left[E_{Q^{*}}\left[X_{t_{n}} 1_{A} \mid \mathcal{F}_{s}\right]\right] \\
& =\limsup _{n \rightarrow \infty} E_{Q^{*}}\left[X_{t_{n}} 1_{A}\right] \\
& =E_{Q^{*}}\left[X_{t} 1_{A}\right] \\
& =E_{Q^{*}}\left[1_{A} E_{Q^{*}}\left[X_{t} \mid \mathcal{F}_{s}\right]\right]
\end{aligned}
$$

where in the first inequality we have made use of Fatou's lemma while in the second equality, Lebesgue's dominated convergence theorem and the right continuity of $X$. Then, $\limsup _{n \rightarrow \infty} E_{Q^{*}}\left[X_{t_{n}} \mid \mathcal{F}_{s}\right] \geq E_{Q^{*}}\left[X_{t} \mid \mathcal{F}_{s}\right]$. Hence $X_{s} \geq E_{Q^{*}}\left[X_{t} \mid \mathcal{F}_{s}\right]$. Now take a sequence $\left\{s_{n}\right\} \subset D$ decreasing to $s \in[0, t)$. Then, by taking a further subsequence if necessary

$$
X_{s}=\lim _{n \rightarrow \infty} X_{s_{n}} \geq \lim _{n \rightarrow \infty} E_{Q^{*}}\left[X_{t} \mid \mathcal{F}_{s_{n}}\right]=E_{Q^{*}}\left[X_{t} \mid \mathcal{F}_{s}\right] .
$$

Now the general case follows from usual localization arguments. Let $\left\{T_{n}\right\}_{n \in \mathbf{N}}$ be a sequence of stopping times increasing to $\mathbb{T}$ such that $X^{T_{n}}$ is bounded. From the previous step there exists $Q_{n} \in \mathcal{Q}$ such that $X^{T_{n}}$ is a $Q_{n}$-supermartingale. We can assume that the sequence $\left\{Q_{n}\right\}$ weakly converges to $Q^{*} \in \mathcal{Q}$ by taking a subsequence if necessary. Now for $\theta$ and $\tau$ stopping times with $\theta \geq \tau$ we have

$$
E_{Q^{*}}\left[X_{\theta}^{T_{n}}-X_{\tau}^{T_{n}}\right]=\lim _{m \rightarrow \infty} E_{Q_{m}}\left[X_{\theta}^{T_{n}}-X_{\tau}^{T_{n}}\right] \leq 0 .
$$

Thus, $X^{T_{n}}$ is a $Q^{*}$-supermartingale.

\section{References}

Aliprantis, C. D. and Border, K. C. Infinite dimensional analysis. A hitchhiker's guide. Springer, Berlin, third edition (2006). ISBN 978-3-540-32696-0; 3-54032696-0. MR2378491.

Bogachev, V. I. Measure theory. Vol. I, II. Springer-Verlag, Berlin (2007). ISBN 978-3-540-34513-8; 3-540-34513-2. MR2267655.

Chen, Z. and Epstein, L. Ambiguity, risk, and asset returns in continuous time. Econometrica, 70 (4), 1403-1443 (2002). MR1929974.

Cheng, X. and Riedel, F. Optimal stopping under ambiguity in continuous time. Math. Financ. Econ., 7 (1), 29-68 (2013). MR3023890.

Delbaen, F. The structure of m-stable sets and in particular of the set of risk neutral measures. In In memoriam Paul-André Meyer: Séminaire de Probabilités XXXIX, volume 1874 of Lecture Notes in Math., pp. 215-258. Springer, Berlin (2006). MR2276899.

Dellacherie, C. Sur l'existence de certains ess.inf et ess.sup de familles de processus mesurables. In Séminaire de Probabilités, XII (Univ. Strasbourg, Strasbourg, 1976/1977), volume 649 of Lecture Notes in Math., pp. 512-514. Springer, Berlin (1978). MR520026.

El Karoui, N. and Quenez, M.-C. Dynamic programming and pricing of contingent claims in an incomplete market. SIAM J. Control Optim., 33 (1), 29-66 (1995). MR1311659.

Ellsberg, D. Risk, ambiguity, and the Savage axioms. Quart. J. Econom., 75 (4), 643-669 (1961). MR3931958. 
Epstein, L. G. and Schneider, M. Recursive multiple-priors. J. Econom. Theory, 113 (1), 1-31 (2003). MR2017864.

Föllmer, H. and Kabanov, Y. M. Optional decomposition and Lagrange multipliers. Finance Stoch., 2 (1), 69-81 (1998). MR1804665.

Föllmer, H. and Kramkov, D. Optional decompositions under constraints. Probab. Theory Related Fields, 109 (1), 1-25 (1997). MR1469917.

Föllmer, H. and Penner, I. Convex risk measures and the dynamics of their penalty functions. Statist. Decisions, 24 (1), 61-96 (2006). MR2323189.

Föllmer, H. and Schied, A. Stochastic finance. An introduction in discrete time, volume 27 of De Gruyter Studies in Mathematics. Walter de Gruyter \& Co., Berlin, extended edition (2004). ISBN 3-11-018346-3. MR2169807.

Goberna, M. A., González, E., Martínez-Legaz, J. E., and Todorov, M. I. Motzkin decomposition of closed convex sets. J. Math. Anal. Appl., 364 (1), 209-221 (2010). MR2576065.

Jacod, J. and Shiryaev, A. N. Limit theorems for stochastic processes, volume 288 of Grundlehren der Mathematischen Wissenschaften [Fundamental Principles of Mathematical Sciences]. Springer-Verlag, Berlin, second edition (2003). ISBN 3-540-43932-3. MR1943877.

Kazamaki, N. Continuous exponential martingales and BMO, volume 1579 of Lecture Notes in Mathematics. Springer-Verlag, Berlin (1994). ISBN 3-540-58042-5. MR1299529.

Kramkov, D. O. Optional decomposition of supermartingales and hedging contingent claims in incomplete security markets. Probab. Theory Related Fields, 105 (4), 459-479 (1996). MR1402653.

Peng, S. Monotonic limit theorem of BSDE and nonlinear decomposition theorem of Doob-Meyer's type. Probab. Theory Related Fields, 113 (4), 473-499 (1999). MR1717527.

Protter, P. E. Stochastic integration and differential equations, volume 21 of Stochastic Modelling and Applied Probability. Springer-Verlag, Berlin (2005). ISBN 3-540-00313-4. MR2273672.

Revuz, D. and Yor, M. Continuous martingales and Brownian motion, volume 293 of Grundlehren der Mathematischen Wissenschaften [Fundamental Principles of Mathematical Sciences]. Springer-Verlag, Berlin, third edition (1999). ISBN 3540-64325-7. MR1725357.

Riedel, F. Optimal stopping with multiple priors. Econometrica, 77 (3), 857-908 (2009). MR2531363.

Rockafellar, R. T. and Wets, R. J.-B. Variational analysis, volume 317 of Grundlehren der Mathematischen Wissenschaften [Fundamental Principles of Mathematical Sciences]. Springer-Verlag, Berlin (1998). ISBN 3-540-62772-3. MR1491362.

Treviño-Aguilar, E. Optimal stopping under model uncertainty and the regularity of lower Snell envelopes. Quant. Finance, 12 (6), 865-871 (2012). MR2930589.

Treviño-Aguilar, E. Semimartingale properties of the lower Snell envelope in optimal stopping under model uncertainty. Braz. J. Probab. Stat., 31 (1), 194-213 (2017). MR3601667.

Treviño-Aguilar, E. The lower Snell envelope of smooth functions: an optional decomposition. Electron. Commun. Probab., 23, Paper No. 12, 10 (2018). MR3771770. 